\title{
Una mirada a la investigación científica en pediatría, en el Paraguay
}

\section{A Glance at Scientific Research in Pediatrics in Paraguay}

\author{
Lourdes Isabel Talavera Toñanez ${ }^{(1)}$
}

\section{RESUMEN}

El presente trabajo tiene como propósito la caracterización de la investigación científica, en el Paraguay. En primer término se realiza una descripción de la misma en la sociedad actual y en América Latina, como asimismo de la práctica médica y la utilización de la ciencia aplicada a la pediatría. La investigación en pediatría es abordada desde los ejes temáticos prevalentes como la salud pública, la infectología, la neurología y la neonatología. Los espacios donde se realizan los estudios son la universidad y los hospitales de la red del Ministerio de Salud Pública y Bienestar Social. Los docentes y los residentes son los investigadores principales. Así también se abordan aspectos relacionados a los medios de publicación de los trabajos científicos de la especialidad. La revista "Pediatría" de la Sociedad Paraguaya de Pediatría, cumple un rol fundamental en la visibilidad de dichos trabajos a nivel nacional y del Cono Sur.

Palabras clave: Investigación en pediatría, publicaciones, producción científica.

\section{INTRODUCCIÓN}

En la actualidad, la sociedad se mueve de manera vertiginosa. La generación de conocimientos es dinámica porque la revolución tecnológica de todo lo vinculado a la informática y la comunicación contribuye a la globalización de los conocimientos. El objetivo de esta revisión es contextualizar la investigación científica en pediatría para presentar posteriormente, en otros números de la revista, aspectos relacionados a la misma.

\begin{abstract}
This article provides an overview of pediatric scientific research in Paraguay. First, we describe the current state of research in today's society and in Latin America, as well as describing contemporary medical practice and the use of science as applied to Pediatrics. Pediatric research is approached from using overarching themes such as public health, infectious diseases, neurology and neonatology. The settings in which the studies were performed are the universities and hospitals that comprise the Ministry of Public Health and Social Welfare Health Network. Faculty and residents are the main investigators. We also review aspects related to the means of publication of scientific papers in the specialty. The "Pediatrics" journal, published by the Paraguayan Pediatric Society, plays a fundamental role in communicating research results at a national and regional level.
\end{abstract}

Keywords: Pediatric research, publications, scientific production.

Emmanuel Kant, describió al hombre como autónomo y auto legislador de sí mismo, es decir un ser heterodoxo. En este tiempo, se lo observa absorbido por el consumo masificado de productos y con la erosión de las identidades sociales, el abandono ideológico y político ${ }^{(1)}$.

En este marco, la razón y la ciencia contribuyen más al consumo masificado, que al uso de la razón

1. Departamento de Investigación de la Universidad Centro Médico Bautista (UCMB). Asunción, Paraguay. E-mail: talaveralou@gmail.com

Recibido: 11/10/2016; Aceptado: 28/11/2015.

Los autores declaran que no existen conflictos de interés en el presente estudio.

http://dx.doi.org/10.18004/ped.2015.diciembre.229-234 
práctica. En consecuencia la barbarie se apoderó de la cultura dando lugar a la intolerancia, al rechazo de parte del hombre a la duda, a la razón. En esta sociedad vertiginosa se inserta una práctica médica influenciada por las características macro sociales y da lugar a separación del médico del alma de sus pacientes $^{(2)}$.

La medicina es una profesión que desarrolló una conexión sistemática entre ciencia y técnica desde la antigüedad. Desde esa época, la enfermedad ha sido un concepto más social que biológico y ha incluido factores que pertenecían a la religión, al derecho o la mitología. La práctica médica utiliza la ciencia de un modo orientado a aplicarla que a crearla. Según Michel Foucault, la medicina como ciencia clínica apareció bajo condiciones que definen con su posibilidad histórica, el dominio de su experiencia y la estructura de su racionalidad. Se acentúan la importancia de la llamada experiencia clínica sobre leyes generales y teorías científicas. La mente clínica prefiere la acción. Esto implica una fuerte tendencia a la improvisación. Los médicos como expertos impondrán su palabra y el poder simbólico de sus puntos de vista sobre aquellos dominados dentro del campo intelectual de la medicina ${ }^{(3-5)}$.

De este modo, la enfermedad es un constructo social y no solamente biológico. En una sociedad de riesgos, los médicos como expertos, definen estilos de vida y por lo tanto, formas de producción y consumo de bienes materiales y simbólicos que cambian los hábitos y costumbres de las personas.

\section{Ciencia, Tecnología y Comunicación}

La ciencia y la tecnología crearon y desarrollaron numerosas explicaciones de gran beneficio para la humanidad. La morbilidad como la mortalidad se modificó a través de los años. La salud se benefició no solo de la biomedicina sino también de otros aspectos que mejoraron la calidad de vida, como por ejemplo: un mejor nivel educativo y cultural de la población, un relativo cuidado del medio ambiente y de los recursos naturales, más oportunidades de empleo y calificación de los recursos humanos; más competitividad de las economías de varías regiones del mundo.

La ciencia no avanzó de modo lineal. Toda su historia está construida sobre discontinuidades, marchas, contramarchas y rupturas epistemológicas. Cada disciplina tiene sus particularidades y hasta la fecha se discute una naturaleza común a toda ciencia. Según Feyerabend las ideas necesitan un tiempo para desarrollar sus ventajas y fortalecerse para sobrevivir incertidumbres. Por otro lado, Khun propone que una realización científica universalmente reconocida por un tiempo, llamada paradigma, proporciona modelos y soluciones a una comunidad científica hasta que aparezca otra y la sustituya. Estos vaivenes, en el desarrollo de la filosofía e historia de la ciencia rigen el comportamiento de los científicos y la producción del conocimiento ${ }^{(6,7)}$.

Aunque el conocimiento científico y tecnológico ha producido aplicaciones de gran beneficio, éstas no se distribuyen equitativamente y ello amplió la brecha entre los países industrializados y aquellos en vías de desarrollo.

En la actualidad, la producción de investigación en los países en desarrollo es de un $10 \%$ y numerosos de esos estudios están financiados y a veces dirigidos por equipos de investigación provenientes de países desarrollados. La investigación científica ha tenido poca prioridad en los políticas de salud en Latinoamérica (LA). En la región, existen pocos grupos de investigación establecidos y el financiamiento local es escaso. A pesar de las barreras, existe una construcción de la necesidad de formar una capacidad local de investigación científica en LA, como un mecanismo de desarrollo para los países ${ }^{(8)}$.

\section{La investigación científica en pediatría}

La investigación en pediatría ha contribuido al progreso del bienestar infantil, en los campos de promoción de la salud, prevención de enfermedades, curación y rehabilitación en los casos necesarios. Sin embargo, los problemas persisten en diferentes órdenes y es preciso que el progreso de la investigación en pediatría continúe para que de este modo se satisfagan las necesidades de los niños, en especial de aquellos afectados por enfermedades prevenibles, en los países pobres ${ }^{(9)}$.

La investigación mejora la práctica clínica habitual al optimizar la formación y estimular el espíritu crítico. A nivel de consultorio ambulatorio o de atención primaria tiene como uno de sus objetivos fundamentales aumentar el conocimiento y evaluar 
la práctica diaria. Las dificultades con las que tropieza tienen relación con la falta de tiempo y de reconocimiento de los gestores: escasez de recursos materiales, estructuras de apoyo e inversión; dispersión de los profesionales; falta de formación metodológica y tradición investigadora; falta de motivación y dificultades para lograr contrato s de investigación y para la publicación de los artículos científicos ${ }^{(10,1)}$.

Asimismo, conviene señalar lo relevado por Castillo et al, acerca de la investigación de estudios clínicos controlados en LA y que incluyó al Paraguay; este equipo refiere que a pesar de algunos países no tienen planes concretos para financiar o establecer focos de investigación locales, los temas más estudiados reflejan que se enfocan aquellos prevalentes en los países como: nutrición, enfermedades respiratorias y las infecciones. En algunos casos, referidos a vacunas se ha contado con el apoyo de la industria farmacéutica. Asimismo la mayor proporción de los estudios clínicos fueron efectuados por profesionales de universidades y fueron publicados en muchos casos en revistas no indexadas en ISI ${ }^{(12-14)}$.

En países del Cono sur, como la Argentina, un estudio menciona que la producción científica estudiada tuvo un 33\% de presentación en congresos y de éstas solamente un 5\% se publicaron. Al evaluar los posibles factores que contribuyen a la presentación y publicación de los trabajos, se identificó a la docencia universitaria que exige que tanto docentes como residentes de pediatría, realicen, presenten o publiquen estudios científicos ${ }^{(15)}$.

Existen numerosas dificultades para publicar en revistas internacionales, las investigaciones realizadas por los equipos científicos de LA. La orientación clínica es la regla porque la formación en las universidades y hospitales responde al área clínica; los docentes en general no tienen formación en ciencia aplicada a la clínica, el manejo del idioma inglés es deficiente y en ocasiones la elección del diseño es inadecuada sin permitir el alcance de resultados aceptables ${ }^{(14,15)}$.

\section{Trabajos científicos y publicaciones}

La situación de la investigación en pediatría, en el país, presenta coincidencias con los de LA y la región. La producción de la investigación científica en pediatría proviene de las universidades y los hospitales. La presentación de los trabajos científicos en los congresos generales, regionales y de las especialidades pediátricas, en numerosos casos se publican en la revista "Pediatría" órgano científico de la Sociedad Paraguaya de Pediatría (SPP).

Según un trabajo publicado por Duarte Caballero et al, se constata durante el periodo estudiado (200220012) de las publicaciones de los artículos científicos en la revista "Pediatría", los temas más tratados fueron: salud pública e infectologia coincidiendo con los de los países de LA, seguidos de neurología y neonatología. Asimismo, en relación a los lugares de realización de las investigaciones, la Universidad Nacional de Asunción, Hospital de Clínicas donde funciona la cátedra de pediatría es uno de los más importantes, como también el Instituto de Investigaciones de la Salud. Por otra parte, los hospitales de las redes del Ministerio de Salud Pública y Bienestar Social como el Pediátrico "Acosta Ñú" y el hospital Nacional de Itauguá, el Instituto de Medicina Tropical y el Hospital General de Barrio Obrero, fueron espacios donde se ejecutaron numerosos estudios científicos. En este periodo también aportó el Instituto de Previsión Social. Llama la atención la escasa o nula producción científica de los entes privados. Este estudio comprendido entre 2002 - 2012, brinda un panorama global acerca de la producción científica en pediatría ${ }^{(16)}$.

Como consecuencia de un proceso de esfuerzo y dedicación de los sucesivos directores de la revista y su consejo editorial, la misma forma parte de la iniciativa SciELO Paraguay (Scientific Electronic Library Online) que es una biblioteca electrónica que abarca una colección seleccionada de revistas científicas que tuvo su origen en SciELO Brasil. Este proyecto está especialmente desarrollado para responder a las necesidades de la comunicación científica en los países en desarrollo, particularmente de LA y el Caribe ${ }^{(17)}$.

El proyecto SciELO Paraguay surge a iniciativa del Consejo Editorial de la revista "Pediatría" e integran esta iniciativa: el Instituto de Ciencias de la Salud, la SPP, la Facultad de Ciencias de la Salud de la Universidad Nacional de Asunción (UNA); el Instituto Nacional de Salud del Ministerio de Salud Pública y Bienestar Social, con el apoyo técnico de la Organización Panamericana de la Salud 
(OPS/OMS). La revista “Pediatría está incluida en los índice Lilacs/ Bireme como asimismo LATINDEX (Sistema Regional de información en línea para registros científicos de América latina, el Caribe, España y Portugal) HINARI (Programa de acceso a la investigación en salud), DIALNET (portal bibliográfico de literatura científica hispana) y DOAJ (Directory of Open Acess Journal) ${ }^{(17)}$.

La incorporación a la iniciativa SciELO Paraguay, favoreció la difusión y la visibilidad de las publicaciones. Los indicadores de producción científica internacional mencionan que LA incrementó la producción y publicación científica en las últimas décadas. Paraguay también avanzó en lo relacionado al registro de la producción bibliográfica de autores paraguayos en bases de datos internacionales ${ }^{(17-19)}$.

A nivel del Cono Sur, la revista "Pediatría" integra el grupo de editores de revistas científicas del Cono Sur. Este grupo de trabajo está integrado por Brasil, Argentina, Paraguay, Uruguay, Chile y Bolivia. Anualmente, los países miembros presentan tres mejores artículos originales y se eligen diez trabajos que son publicados en las revistas de dichos países. Esta es una actividad promovida por el grupo en la búsqueda de la difusión y reconocimiento de los mejores artículos origínales de pediatría, en el Cono Sur ${ }^{(20)}$.

\section{PROCIENCIA - CONACYT}

El Consejo Nacional de Ciencia y Tecnología (CONACYT), a partir de 2013, lleva adelante el Programa Paraguayo para el Desarrollo de la Ciencia y Tecnología (Prociencia), cuyo objetivo es fortalecer las capacidades nacionales para la investigación científica y desarrollo tecnológico, de modo a contribuir con el aumento de la capacidad productiva, la competitividad y mejorar las condiciones de vida en el Paraguay ${ }^{(21)}$.

Este programa busca focalizar acciones en el desarrollo de capacidad nacional, preservado la visión de sistema y de procesos correspondiente al sector de ciencia, tecnología e innovación. Convocó a la presentación de proyectos en el año 2014. De la lista de adjudicados por el Componente I. Fomento de la investigación científica, para el año 2014, y la que se conoció en marzo de este año, se viene actualizando desde entonces, fueron seleccionados un grupo interesante de proyectos de investigación en pediatría, que están financiados con recursos del Fondo para la Excelencia de la Educación y la Investigación (FONACIDE), tabla $\mathbf{1}^{(22,23)}$.

Tabla 1. Proyectos de investigación en pediatría, adjudicados por Prociencia, años 2014- 2015.

\begin{tabular}{|c|c|c|c|}
\hline Año & Institución & Nombre del proyecto & Área \\
\hline 2014 & $\begin{array}{l}\text { Instituto de Investi- } \\
\text { gaciones en Ciencias } \\
\text { de la salud-Univer- } \\
\text { sidad Nacional de } \\
\text { Asunción/IICS-UNA }\end{array}$ & $\begin{array}{l}\text { Análisis Multi-locus de secuencias } \\
\text { repetitivas en tándem de Número } \\
\text { variable (MLVA) y detección de } \\
\text { genes que codifican factores de } \\
\text { virulencia de Staphylucoccus aureus } \\
\text { aislados de niños paraguayos }\end{array}$ & $\begin{array}{l}\text { Ciencias } \\
\text { Médicas y } \\
\text { de la salud }\end{array}$ \\
\hline 2014 & $\begin{array}{l}\text { Centro Multidisci- } \\
\text { plinario de Investi- } \\
\text { gación Tecnológicas } \\
\text { CEMIT - UNA }\end{array}$ & $\begin{array}{l}\text { Estado nutricional, respuesta } \\
\text { inflamatoria de la microbiótica } \\
\text { intestinal de niños en edad escolar } \\
\text { con poliparasitosis }\end{array}$ & $\begin{array}{l}\text { Ciencias } \\
\text { Médicas y } \\
\text { de la salud }\end{array}$ \\
\hline 2015 & $\begin{array}{l}\text { Instituto de } \\
\text { Desarrollo }\end{array}$ & $\begin{array}{l}\text { Prevalencia nacional de anemia en } \\
\text { lactantes de } 6 \text { a } 24 \text { meses de edad }\end{array}$ & $\begin{array}{l}\text { Ciencias Médi- } \\
\text { cas y de la saluo }\end{array}$ \\
\hline 2015 & $\begin{array}{l}\text { Asociación de fun- } \\
\text { cionarios de la } \\
\text { Universidad Nacio- } \\
\text { nal de Caaguazú }\end{array}$ & $\begin{array}{l}\text { Evaluación del volumen y calidad } \\
\text { de los líquidos consumidos por los } \\
\text { niños/as y adolescentes de Asunción, } \\
\text { central y Caaguazú y la asociación con } \\
\text { el sobrepeso y la obesidad en niños/as } \\
\text { y adolescentes en el año } 2014\end{array}$ & $\begin{array}{l}\text { Ciencias } \\
\text { Médicas y } \\
\text { de la salud }\end{array}$ \\
\hline 2015 & $\begin{array}{l}\text { Hospital General Pediá- } \\
\text { trico Niños de Acosta } \\
\text { Nú. Ministerio de } \\
\text { Salud Pública y Bienes- } \\
\text { tar Social -MSP y BS }\end{array}$ & $\begin{array}{l}\text { Exposición prenatal a pesticidas: } \\
\text { efectos adversos neonatales y del } \\
\text { neurodesarrollo a los } 18 \text { meses de } \\
\text { edad. Un estudio de cohortes } \\
\text { prospectivo. }\end{array}$ & $\begin{array}{l}\text { Ciencias } \\
\text { Médicas y } \\
\text { de la salud }\end{array}$ \\
\hline 2015 & $\begin{array}{l}\text { Fundación Renaci - } \\
\text { Red Nacional de } \\
\text { Atención del } \\
\text { cáncer infantil }\end{array}$ & $\begin{array}{l}\text { Determinación de la enfermedad } \\
\text { residual mínima como factor } \\
\text { pronóstico para la estratificación de } \\
\text { riesgo en las leucemias linfoblásticas } \\
\text { agudas. }\end{array}$ & $\begin{array}{l}\text { Ciencias } \\
\text { Médicas y } \\
\text { de la salud }\end{array}$ \\
\hline 2015 & $\begin{array}{l}\text { Fundación Renaci - Red } \\
\text { Nacional de Atención } \\
\text { del cáncer infantil }\end{array}$ & $\begin{array}{l}\text { Estudio de marcadores moleculares } \\
\text { como factores pronósticos en } \\
\text { leucemias infantiles. }\end{array}$ & $\begin{array}{l}\text { Ciencias } \\
\text { Médicas y } \\
\text { de la salud }\end{array}$ \\
\hline 2015 & $\begin{array}{l}\text { Universidad } \\
\text { Nacional de } \\
\text { Itapuá }\end{array}$ & $\begin{array}{l}\text { Análisis de la influencia del programa } \\
\text { consultorio de crecimiento y desarrollo, } \\
\text { en la salud de los niños menores de } 2 \\
\text { años del Hospital Regional de } \\
\text { encarnación }\end{array}$ & $\begin{array}{l}\text { Ciencias } \\
\text { Médicas y } \\
\text { de la salud }\end{array}$ \\
\hline 2015 & $\begin{array}{l}\text { Universidad } \\
\text { Católica }\end{array}$ & $\begin{array}{l}\text { Adolescencia prevenida: detección de } \\
\text { indicadores de riesgo - resiliencia para } \\
\text { la educación de calidad de vida, } \\
\text { empoderamiento de monitores del } \\
\text { Territorio social beneficiario y } \\
\text { Formación de estudiantes en Investi- } \\
\text { gación Aplicada Transcultural. }\end{array}$ & $\begin{array}{l}\text { Estructuras y } \\
\text { Relaciones } \\
\text { Sociales }\end{array}$ \\
\hline
\end{tabular}

Es interesante encontrar entre los proyectos de investigación adjudicados con financiamiento a través del CONACYT que éstos incursionan en estudios biomoleculares, epidemiológicos y sociales. Este abanico de posibilidades permite que los investigadores puedan realizar investigaciones con enfoques cualitativos como cuantitativos. Inicialmente los procedimientos cuantitativos y sus técnicas marcaron un predominio casi exclusivo de la producción científica pero actualmente se emplean los cualitativos solos o integrados a los cuantitativos. Esta imbricación permite resultados y conclusiones más abarcantes y rigurosas. En el campo de la pediatría los factores del entorno influencian de manera contundente el proceso de salud-enfermedad, del niño ${ }^{(24-27)}$ 
En relación a los estudios biomédicos es de vital importancia contar con criterios certeros que establezcan marcos conceptuales basados en la bioética para su realización como asimismo una definición clara del problema a investigar para con seguridad seguir adecuadamente los pasos posteriores del diseño y arribar a conclusiones que constituyan una contribución original al conocimiento ${ }^{(28-30)}$.

\section{CONCLUSIÓN}

A manera de conclusión se puede expresar que las actividades de investigación en pediatría tiene un mayor énfasis a nivel académico, en la universidad como tarea de docentes y residentes. La existencia de

\section{REFERENCIAS}

1. Kant E. Crítica de la razón pura. México: Porrúa; 1996.

2. Díaz A. La medicina desalmada. Montevideo: Trilce; 2004.

3. Foucault M. El nacimiento de la clínica: una arqueología de la mirada médica. Barcelona: Paidós; 2010.

4. Foucault M. La hermenéutica del sujeto. La Plata: Editorial Altimira; 2012.

5. Portillo J. Reflexiones acerca de la relación médico-empresas farmacéuticas. Rev Médica del Uruguay. 1998;14:60-68.

6. Feyerabend P. La conquista de la abundancia: la abstracción frente a la riqueza del ser. Barcelona: Paidós; 2013.

7. Kuhn TS. La estructura de las revoluciones científicas. México: Fondo de cultura Económica; 2006.

8. Castillo C, Pleticosik X, Pizarro F. Estudios clínicos controlados aleatoreos en la pediatría de Latinoamérica (1996-2005). Rev Chil Pediatr. 2009;80(5):420-26.

9. Working Group on Women and Chile Health. Improving child health: the role of research. BMJ. 2002(324):1444-47.

10. Diez D. Investigaciones en pediatría de atención primaria: la asignatura pendiente. Anales de Pediatría, Barcelona. 2004(61):289-91. obstáculos se relaciona con un inadecuado manejo de las herramientas metodológicas, el idioma inglés y la informática. También la escasa accesibilidad, a fuentes sostenibles de financiamiento dificulta el desarrollo de las investigaciones en pediatría, aunque es esperanzador el rol de Prociencia del CONACYT. Sin embargo, la fortaleza de la investigación, en el campo de la pediatría radica hasta la fecha en la revista de la SPP, que brinda un espacio invaluable para la publicación de los trabajos científicos y la posibilidad de socializarlos, en el Cono Sur. El compromiso de la SPP está en su papel formador al ofrecer alternativas para superar las dificultades en la realización rigurosa de las investigaciones en Pediatría.

11. Durán P. La importancia de un buen comienzo: definición del problema de investigación. Arch Argent Pediatr. 2005;103(1):91-92.

12. Cardoso P, Calabró P. Investigación clínica farmacológica en pediatría: ¿es ético y legal experimentar en niños?. Arch Argent Pediatr. 2005;103(1): 46-50.

13. Smith R. Publishing research from developing countries. Stat Med. 2002;21:2869-77.

14. Paraje G, Sadana R, Karam G. Public health: incresing international gaps in health-related publications. Science. 2005;308:959-60.

15. Majurin M, Cutri A, Torres F, Noguerol M, Ossorio M, Durán P, Ferrero F. Evaluación de la producción científica en la residencia de pediatría. Arch Argent Pediatr. 2009;197(1):26-29.

16. Duarte Caballero A, Fonseca R, Espínola de Canata M, Duarte Masi S. Productividad de la revista Pediatría periodo 2002-2012. Pediatr (Asunción). 2014;41(1):9-14.

17. Proyecto Scielo Paraguay. Pediatr (Asunción). 2005;32(1):9.

18. Galeano ME, Parra G. Productividad científica del Paraguay en el área de Biomedicina: un análisis bibliométrico. Mem Inst Invest Cienc Salud. 2007;5(1):26-30.

19. Consejo Nacional de Ciencia y Tecnología. Indicadores de Ciencia y Tecnología en Paraguay. 4ta edición. Asunción: Conacyt; 2012. 
20. Pantoja Ludeña M, Mazzi E. Reseña histórica del grupo de editores del Cono Sur. Rev Chil Pediatr. 2003;7(6):609-10.

21. CONACYT. Programas y proyectos, Prociencia [Internet]. Asunción: CONACYT [citado 27 de nov 2015]. Disponible en: http://www.conacyt.gov.py/programaprociencia

22. CONACYT. Nómina de proyectos de investigación adjudicados, Resolución n ${ }^{\circ} 437$ de 2014 [Internet]. Asunción: CONACYT [citado 27 de nov 2015]. Disponible en: http://www.conacyt.gov.py/nomina-proyectosadjudicados-prociencia

23. CONACYT. Nómina de proyectos de investigación adjudicados, Resolución no 095 de 2015 [Internet]. Asunción: CONACYT [citado 27 de nov 2015]. Disponible en: http://www.conacyt.gov.py/nomina-proyectos-deinvestigación-adjudicados/resolución-n095-2015

24. Tamayo y Tamayo M. El proceso de la investigación científica. México: Limusa; 2013.
25. Guttantin F. Investigación cualitativa interpretativa: una caja de herramientas. Asunción: Centro de estudios Antropológicos de la Universidad Católica (CEADUC); 2012.

26. Habermas J. La lógica de las ciencias sociales. Madrid: Tecnos; 1996.

27. Taylor S. Introducción a los métodos cualitativos de investigación. Barcelona: Paidós; 1994.

28. Durán P. La importancia de un buen comienzo: definición del problema en estudios de investigación. Arch Argent Pediatr. 2005;103(1):91-92.

29. Marí E. Elementos de epistemología comparada. Buenos Aires: Puntosur; 1991.

30. Rivera S. Alcances y límites del modelo deontológico en el campo de la investigación biomédica. Arch Pediatr. 2009;107(1);43-48. 